\title{
Energy-Efficient Medium Access for Wireless Sensor Networks under Slow Fading Conditions
}

\author{
Changmian Wang \\ IET Department \\ NTNU \\ Trondheim, Norway \\ Email: changmia@iet.ntnu.no
}

\author{
Liuguo Yin \\ School of Aerospace \\ Tsinghua University \\ Beijing, China \\ Email: yinlg@tsinghua.edu.cn
}

\author{
Geir E. Øien \\ IET Department \\ NTNU \\ Trondheim, Norway \\ Email: oien@iet.ntnu.no
}

\begin{abstract}
In this work, we first study and then propose an optimal version on the medium access control mechanism known as carrier sense multiple access with collision avoidance (CSMA/CA). This protocol is adopted by many wireless communication standards, such as the IEEE 802.11 series and IEEE 802.15.4 (ZigBee). We first identify its drawbacks in a slow fading channel environment, and show that the CSMA/CA can be very energy-inefficient, especially in many wireless sensor network (WSN) applications where the traffic demand is low. In particular, we study communication over a Rayleigh fading channel, which we model by means of a time invariant Markov process. We then show that the decision regarding the optimal access time on such a channel can be formulated as an optimization problem, and that an optimal solution can be accurately derived. We also simulate a ZigBee node in a Rayleigh fading environment and the result shows that optimum channel access can save significant amount of energy compared to conventional CSMA/CA.
\end{abstract}

\section{INTRODUCTION}

In recent years, much research has been devoted to wireless sensor networks (WSNs). Their unique features include low power, low energy cost, and small node size, which make such networks very different from typical mobile networks. Some challenges were identified by researchers (see e.g., [1], [2]). One of the main concerns when designing such networks is how to operate the nodes in an energy-efficient way. As was pointed out by the authors in [2], true energy efficiency can only be achieved by cross-layer design.

The most widely adopted medium access control (MAC) method is probably the one that is implemented in the IEEE 802.11 family for wireless local networks (WLAN), namely the CSMA/CA method, where CSMA stands for carrier sense multiple access and CA stands for collision avoidance [3]. This collision avoidance method was also adopted by the IEEE 802.15.4 standards (i.e., ZigBee), which was designed for low rate, low cost applications, such as in many WSN applications.

An important tool employed by CSMA/CA to avoid potential collisions is the random backoff mechanism. In particular, CSMA/CA chooses a random backoff number between 0 and the contention window (CW) length, and use this random number multiplied with one unit backoff time to get a random time delay before transmission. Such randomness in time among different nodes can lower the probability of simultaneous transmissions. In addition, under certain cases, $\mathrm{CW}$ value can be exponentially increased until it reaches a maximum. For example in IEEE 802.11, any unsuccessful transmission of a frame will lead to an CW increase [4], while the CW increment in IEEE 802.15.4 is a result of a negative clear channel assessment (CCA) [5], or in other words, by a sensed busy channel. Despite the difference, the random backoff mechanism is able to perform its duty well, and the probability of frame collisions is reasonably reduced with its help.

In a WLAN, the traffic demand is usually high, thus how to maximize an individual node's throughput and avoid packet collision with the others is one of the central concerns. The fact that CSMA/CA has been so widely adopted seems to indicate it works fairly well in practice. However, in a variety of different WSN applications, nodes are mostly driven by a battery with limited energy, and designed to operate for a long time period. Given its fixed total quantity of energy, the total number of information bits a node could convey is limited. To save energy, most of the sensor nodes therefore enter a hibernation state whenever possible, which is usually termed as long duty cycle. The difference in the throughput demand naturally corresponds to a low traffic density property for WSN applications, rather than the usual high traffic assumption in regular communication networks. Such property also has a direct consequence when it comes to using CSMA/CA as channel access scheme. In contrast to IEEE 802.11, a node does not sense the channel during the backoff countdown phase in IEEE 802.15.4, rather the channel can only be sensed by performing CCA, which follows immediately after the backoff counter reached 0 [5], [6]. The execution of CCA takes 8 symbol time [5], which is at most $0.4 \mathrm{~ms}$. Compared it with the long duty cycle a WSN node often has, and keep in mind that the low traffic density property, it is foreseeable, and we also assume this throughout this work that a node has almost a negligible probability of sensing a busy channel, i.e. a node can always start a transmission with little channel access contention. The above assumptions are reasonable and valid for many application scenarios, for example it could be the medical sensors placed on human body reporting the measurements sporadically to a center node, or could be some event-driven sensor nodes monitoring rarely happened event (such as fire, gas leaking, etc.). However, the real question is: would CSMA/CA still be the right choice for WSNs, given that 
it was originally designed for high traffic demanding networks and frame collision is the main obstacle to the performance?

Taking the unslotted CSMA/CA specified by ZigBee as an example (introductions on unslotted CSMA/CA can be found for example in [7]), if an acknowledgement (ACK) is required upon an transmission frame, the sender will wait a certain time (The ACK Timeout time) before it infers that a failure has occurred. If this is the case it either resorts to CSMA/CA once more for a retransmission, or it discards the frame if the maximum number of retries has been reached. For the former case, we can define the time span of one such iteration in the retransmissions, as one attempt time:

$$
\begin{aligned}
\text { one attempt time }= & \text { backoff time }+ \text { transmission time } \\
& + \text { ACK timeout time }
\end{aligned}
$$

where the backoff time is given by:

$$
\text { backoff time }=\text { number of backoffs } \times \text { aUnitBackoffPeriod }
$$

where "aUnitBackoffPeriod" is a parameter specifies the time for a single backoff operation. The number of backoffs is chosen uniformly from the interval $\left[0,2^{\mathrm{BE}}-1\right]$, where $\mathrm{BE}$ is the backoff exponent, and the transmission time is very small, usually much less than 1 symbol time. The default values used in ZigBee can be found in Table $I$ in the Appendix.

ZigBee can operate in 3 different frequency bands. If we assume that a node operates at $f_{c}=868 \mathrm{MHz}$ with a bit rate of $20 \mathrm{Kbps}$, and that the relative velocity between two nodes is $v=0.2 \mathrm{~m} / \mathrm{s}$, the channel coherence time can be roughly estimated to be: $T_{c} \approx 1 / f_{m}=1.728 \mathrm{~s}$, where $f_{m}=v / \lambda$ is the maximum Doppler shift. If we assume one frame length to be 66 bytes, including 6 bytes length of packet head by default ${ }^{1}$, and also assume that the random backoff always reaches its maximum, we can use (1) to conclude that one attempt time is upper bounded by $0.0394 \mathrm{~s}$. With a maximum of 3 retries, the sender will discard the packet within 0.1182 seconds, much smaller than $1.728 \mathrm{~s}$. We can see clearly that in case a failure is caused by a deep fade, triple transmission energy plus the circuit energy will under our assumption of low traffic demand be wasted by using CSMA/CA. In low traffic density low mobility networks, slowly varying deep fades are in fact most likely the main reason for transmission failures. A waste of energy such as demonstrated here can therefore be a serious problem in such scenarios, prohibiting the long time operation goal for sensor nodes. Therefore, in this work, we study this case in detail, and try to combine the physical layer information and MAC layer in a crosslayer design. Specifically, we use a time invariant Markov process to model the slow fading channel, and we also study the stationary distribution and channel state evolution of this model. Then we formulate the channel access problem as an optimization problem, and derive the optimal access time by solving this problem. We run simulations to compare the

\footnotetext{
${ }^{1}$ Actually the pay load length in a ZigBee frame is a variable, ranging from $8-128$ bytes; however 60 bytes is enough for many WSN applications.
}
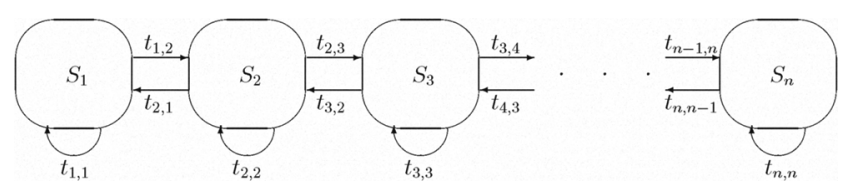

Fig. 1. A $n$-state Markov chain modeling Rayleigh Fading Channel.

energy savings between the optimal scheme and CSMA/CA, and provide explanations wherever needed.

The remainder of this paper is organized as follows. In Section II, we establish the Markov model for the Rayleigh fading channel. In Section III we formulate the optimization problem and analyze the simulation results. Finally, we draw conclusions in Section IV.

\section{MARKov Modeling OF RAYLEIGH FADING CHANNELS}

\section{A. A Time-Invariant Markov Channel Model}

In contrast to the mobile networks where handling a phone call from a fast moving vehicle can be crucial, in this work, we focus more on the networks with slow velocity, which is wellsuited for many WSN applications, such as those industrial process monitoring applications, event detection applications, etc. Such low mobility naturally leads to a small Doppler shift, which corresponds to a slow fading channel. In [8], the authors showed that a time-invariant Markov process is a good approximation of the real channel, especially when the Doppler shift is small. In addition, such a model can also capture the correlation of the fading amplitude between consecutive data symbols. Specifically, the authors model the Rayleigh fading channel by a time invariant finite state Markov process $\left\{X_{t}\right\}_{t=0}^{\infty}$, with state space denoted by $\mathcal{S}=$ $\left\{S_{1}, S_{2}, \ldots, S_{n}\right\}$. In particular, each fading state can only transit to one of two neighboring states as is depicted in Fig. 1.The transition probability between two states is then given by the probability transition matrix $\mathbf{T}$, with its entry $t_{i j}$ denoting the transition probability from the current state $i$ to the next state $j: t_{i, j}=\operatorname{Pr}\left(X_{t+1}=S_{j} \mid X_{t}=S_{i}\right)$. In our case, the transition matrix $\mathbf{T}$ is thus a tridiagonal matrix. Although the authors in [8] didn't mention this, but for such structure in matrix $\mathbf{T}$, it can be proved that $\lim _{k \rightarrow \infty} \mathbf{T}^{k}$ exists ${ }^{2}$ [9], where $k$ denotes the number of matrix transitions. This limit means that, for an arbitrary initial distribution vector, denoted by $\mathbf{e}^{T}(0)=\left(e_{1}(0), e_{2}(0), \ldots, e_{n}(0)\right)$, the $k^{\text {th }}$ step probability distribution vector, which is defined as

$$
\mathbf{e}^{T}(k)=\mathbf{e}^{T}(0) \mathbf{T}^{k}
$$

will surely converge as $k \rightarrow \infty$. In other words, the probability of being in each state in the long run is determined, regardless of the initial state.

\footnotetext{
${ }^{2}$ Due to page number limitations, the proof and further in-depth discussions can be found in the journal version of this work, or can be acquired from the authors by email.
} 


\section{B. The Channel Evaluation Based on Markov Chain Modeling}

As it has been discussed in the introduction part that CSMA/CA takes little consideration on the underlying physical channel conditions, since the design goal is to overcome the frame collisions, which is usually assumed to be the main obstacle to the performance in regular communication networks. However, as we have also argued in the above, for a mobile sensor node with little demand on the throughput, the fading prone, time variant wireless channel can actually be the main obstacle to performance. The above channel modeling by means of a time-invariant Markov chain is just such a tool which can enable us to evaluate and even predict the channel behavior. Later we will see that such channel evaluations and predictions can help us to gain insight in designing the optimum channel access scheme in accordance with the channel condition.

For a sensor node, at the moment that it starts the transmission, the channel might be in different states. However, during the time interval from the transmission starts until it completes, the channel also varies or devates from the starting state. Fig. 2 and Fig. 3 depict an example of the channel evolution described above. We partition the received SNR on a Rayleigh fading channel into 5 consecutive regions, each region corresponding to one channel state. The boundary values separating these regions are chosen to be the SNRs corresponding to a BER of $10^{-1}, 10^{-2}, 10^{-3}$, and $10^{-4}$ respectively, under the assumption of BPSK being the designated modulation scheme. So for example, at state 3 , then the received SNR is in the region corresponding to a BER between $10^{-2}$ and $10^{-3}$.

In Fig. 2, we simulated the system performance under the speed of $1 \mathrm{~m} / \mathrm{s}$, with average received SNR $\bar{\gamma}=5 \mathrm{~dB}$. We choose the initial state of the left plot to be $\mathbf{e}_{1}=[1,0,0,0,0]^{T}$, which means that the received SNR is in region 1 when transmission starts. The plot on the right shows the evolution from an initial vector $\mathbf{e}_{3}=[0,0,1,0,0]^{T}$. Both plots show a gradual convergence from the initial to the stationary state as time proceeds. They also show that the speed of convergence is different depending on the initial state, even though they will eventually converge to the same distribution.

In Fig. 3. we again simulate probability evolution for the initial vector $\mathbf{e}_{3}$, i.e. state 3 , but with lower speed $v=0.2$ $\mathrm{m} / \mathrm{s}$ and different values of $\bar{\gamma}$. Compare both plots in Fig. 3 we can see that changes in $\bar{\gamma}$ affect the convergence speed little, but clearly affect the stationary distribution. Comparing the left plot in Fig. 3 and the right plot in Fig. 2, we can also see that the lower the speed is, the slower it converges. The results match our intuition, since lower mobility implies a higher temporal correlation between channel states.

It is important to note that the convergence speed can in fact be crucial to the performance of a given MAC protocol, and thus should be taken into account in the MAC design. As we showed earlier, if a ZigBee node operates in the $868 \mathrm{MHz}$ frequency band, and with 3 retries, the total time spent on the maximum allowed number of retries before the packet must be discarded is upper bounded by 0.1182 seconds. However, as
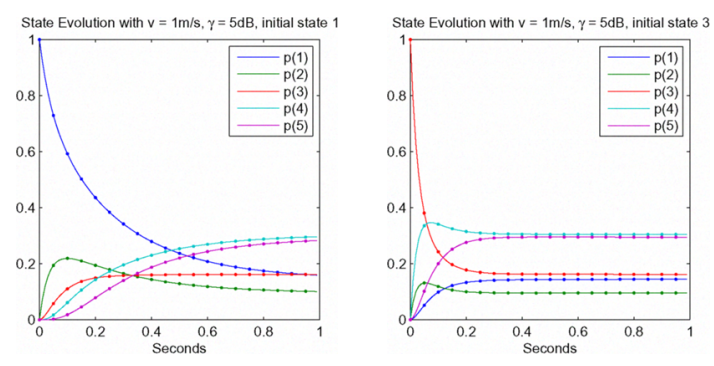

Fig. 2. Probability evolution under $v=1 \mathrm{~m} / \mathrm{s}, \bar{\gamma}=5 \mathrm{~dB}$.
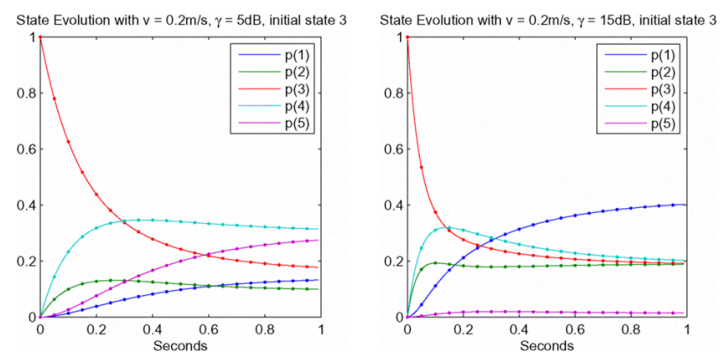

Fig. 3. Probability evolution under $v=0.2 \mathrm{~m} / \mathrm{s}$ and $\bar{\gamma}=5 \mathrm{~dB}$ and $\bar{\gamma}=15$ dB respectively.

the left plot in Fig. 2 shows, if the current state is $\mathbf{e}_{1}$, even after 0.1182 seconds, the channel still has a very high probability of staying in the deep fading state. It implies that retransmissions defined by the protocol don't improve the successful rate at all and it is almost always just a waste of both transmission energy and circuit energy, which has been shown to be as important in short range wireless communications [10]. With maximum 3 retries and assuming negligible probability of the channel being occupied, this would mean that triple energy is wasted without any success. Therefore, it is important to know when to start a transmission that can be successful and at the same time consume the least energy. We shall explore this question in the next section.

\section{Cost Function Definition AND A Proposed Optimal Channel ACCESS Mechanism}

\section{A. Cost Function and Optimal Channel Access Definitions}

To find the optimal channel access time, first we need to define a cost function, which should reflect our desired objective to reduce the energy consumption under physical channel condition as the main obstacle. In this work, we define the cost function to be the minimal expected energy ${ }^{3}$ to complete one frame transmission successfully.

To accomplish the definition of cost function, we first assume perfect channel knowledge in the sense that the

\footnotetext{
${ }^{3}$ Strictly speaking, the energy should be the cost multiplied by a constant, however, for the optimization point of view such constant will not affect the optimal result.
} 
transmitter knows the current channel state in terms of SNR of the receiver side when the transmitter acquires the channel (in IEEE 802.15.4 acquiring the channel means that the backoff counter has reached 0 and CCA result is positive, so that a transmission is eligible to start.). We also assume that the node's velocity is given, so that the channel transition matrix $\mathbf{T}$ can be estimated and we use the Markov model presented in the above section to predict future channel behavior. As discussed in subsection II-A, for a given arbitrary initial state vector, we can derive the $k^{\text {th }}$ step distribution vector according to (3). We define the channel state distribution function $\mathbf{f}_{i}^{T}(k)$ to be the $k^{\text {th }}$ step distribution vector for an initial state $\mathbf{e}_{i}$ :

$$
\begin{aligned}
\mathbf{f}_{i}^{T}(k) & =\mathbf{e}_{i}^{T} \mathbf{T}^{k} \\
& =\sum_{l=1}^{n} \lambda_{l}^{k} \mathbf{P}_{i l} \mathbf{P}_{l *}^{-1}
\end{aligned}
$$

where we decompose the matrix $\mathbf{T}$ by using an eigenvalue decomposition, $\lambda$, denotes the eigenvalues, $\mathbf{P}$ is the eigenvector matrix, indices $i l$ denotes the element in $i^{\text {th }}$ row and $l^{\text {th }}$ column, and indices $l *$ denotes the $l^{\text {th }}$ row. We further define the expected cost sequence for initial state $\mathbf{e}_{i}$ to be:

$$
l_{i}(k)=\mathbf{w}^{T} \mathbf{f}_{i}(k)
$$

where $\mathbf{w}^{T}=\left(w_{1}, \ldots, w_{n}\right)$ is a cost vector. This vector can be interpreted as the necessary transmission power that has to be used for different states to reach a certain target state. Besides the channel condition can vary during the transmission, which will result to variations in expected cost sequences accordingly. Assume the transmission of one frame takes $L$ units of channel transitions, then the cost function which is defined to be the minimal expected total energy to finish one frame transmission successfully, should be given by the sum of $L$ cost sequences:

$$
\begin{aligned}
\sum_{k}^{k+L} l_{i}(k) & =\sum_{k}^{k+L} \mathbf{w}^{T} \mathbf{f}_{i}(k) \\
& =\sum_{k}^{k+L} \sum_{l=1}^{n} \sum_{m=1}^{n} \lambda_{l}^{k} \mathbf{P}_{i l} w_{m} \mathbf{P}_{l m}^{-1} .
\end{aligned}
$$

Noted that $k$ means that the actual transmission starts $k$ channel transition time after the transmitter has acquired the channel.

Upon defining the cost function, we thus define the optimum channel access time to be the optimized value of $k$ that can minimize the cost function. In other words, we would like to find the optimal channel access time such that the subsequent transmission has the minimum total cost. With our definitions, the problem can now be formulated as the following optimization problem:

$$
k_{i}^{*}=\arg \min _{k} \sum_{k}^{k+L} l_{i}(k) .
$$

where $i$ implies that the initial state is given by $\mathbf{e}_{i}$.

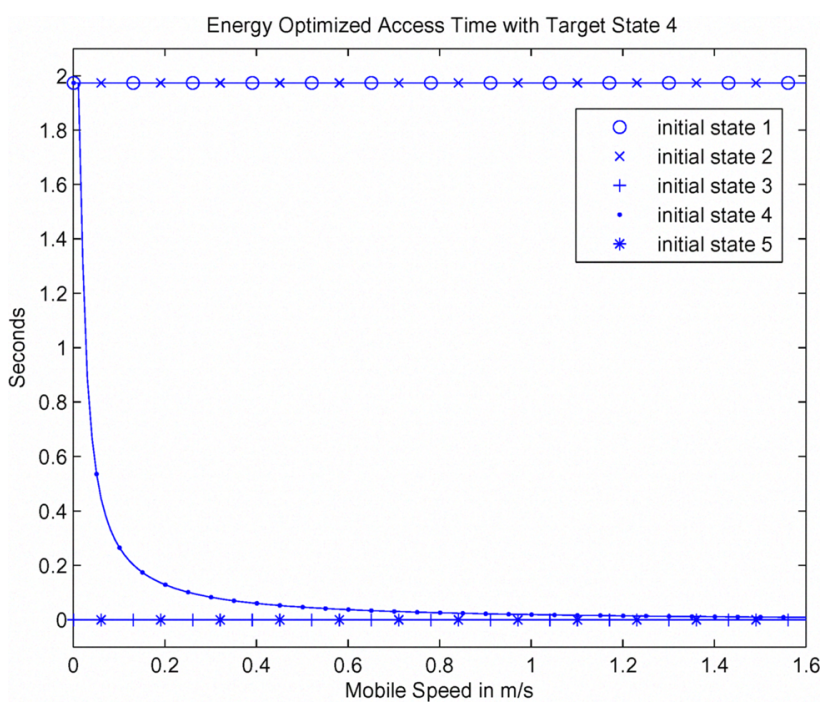

Fig. 4. The Optimized Channel Access Time with Target Channel State 4.

\section{B. Simulations and Discussion}

In this subsection, we will evaluate and discuss our proposed optimum access method in a Rayleigh fading channel. In particular, we will first find out the optimal channel access time for different initial channel states under different channel transition matrix Ts. Then we will compare the energy performance between the optimal channel access time and those given by the CSMA/CA specified in IEEE 802.15.4. We apply the exact estimation method proposed in [8] to acquire the probability transition matrix $\mathbf{T}$.

The weight vector assignment is done according to the region boundaries and the target SNR intended to achieve. The weight vector is defined by us to be:

$$
\mathbf{w}=\left(\frac{\mathrm{SNR}_{\mathrm{tar}}}{\mathrm{SNR}_{1}}, \frac{\mathrm{SNR}_{\mathrm{tar}}}{\mathrm{SNR}_{2}}, \cdots, \frac{\mathrm{SNR}_{\mathrm{tar}}}{\mathrm{SNR}_{n}}\right)
$$

where the $\mathrm{SNR}_{\mathrm{tar}}$ denotes the required SNR at target state and $\mathrm{SNR}_{i}$ denotes the SNR at state region boundaries. With this definition, we normalize the required power for different states to reach the target state: I.e. when we are at target state, it takes 1 unit power to transmit. This definition also has the advantage that it can be computed without any information on the actual channel gain, this is because all transmissions experience the same channel, the extra power the transmitter needs to adjust in order to complete the transmission successfully (reach the target state) is proportionally given by the elements in $\mathbf{w}$. However, since we only have 4 boundaries but 5 different states, we shall assume the last state has an BER of $10^{-5}$. As an example, assuming a target state 4 , the weight vector can be computed to be $\mathbf{w}=[8.42,2.56,1.45,1.00,0.1901]^{T}$.

In addition, IEEE 802.15.4 totally supports 3 different transmission rates, which are $20 \mathrm{ksymbol} / \mathrm{s}$ at $868 \mathrm{MHz}, 40$ $\mathrm{ksymbol} / \mathrm{s}$ at $902 \mathrm{MHz}$, and $62.5 \mathrm{ksymbol} / \mathrm{s}$ at $2450 \mathrm{MHz}$ respectively. Given the fact that only low rate transmissions are supported by IEEE 802.15.4, it is not hard to see most 
of the transmissions will experience a flat fading channel. Therefore, we simulate the node moves at different velocities in a Rayleigh flat fading channel and we set the average received SNR $\bar{\gamma}=5 \mathrm{~dB}$.

In practice, there is usually a time constraint from the higher communication protocol layer upon the transmission of each frame. Hence for the MAC layer it implies that the node has to access the channel within a given time constraint, i.e. it is often required that $t<t_{\text {lim }}$. This in our case can be translated into a limit on the maximum number of allowed channel state transitions, i.e. we require $k<k_{\mathrm{lim}}$. Therefore, the problem of (9) should really be modified into the following constrained optimization problem:

$$
k_{i}^{*}=\arg \min _{k<k_{\lim }} \sum_{k}^{k+L} l_{i}(k)
$$

In our simulations, we specially assume that each frame has to be transmitted within a hard threshold of 2 seconds, i.e. $t_{\mathrm{lim}}=1.9736$ seconds given that the frame length is 66 bytes and it takes 0.0264 seconds to accomplish the transmission. Strictly speaking, this is an integer programming problem, since $k$ can only be positive integers. However, according to [8], each channel transition corresponds to one channel symbol time. In ZigBee at $868 \mathrm{MHz}$ a direct sequence spread spectrum (DSSS) technique is used for transmission, so that each symbol is further spread into 15 chips and to be transmitted by BPSK modulation. With a symbol rate of $20 \mathrm{Kbps}$ at this frequency, one transition time only corresponds to $10^{-6}$ seconds. Therefore, treating $k$ as a continuous variable and employing a round operation after optimization can definitely give enough accuracy in time.

Fig. 4 shows the optimized channel access time in terms of minimum expected energy consumption, for various initial states. One feature is that except for the initial state 4 , i.e. $\mathbf{e}_{4}$, the optimal channel access time for the rest of the initial states tend to have a binary decision, i.e. either to start the transmission immediately upon acquiring the channel, or it is best to delay the transmission as long as possible until the channel distribution becomes stationary. It is understandable that the node starts accessing the channel immediately if the current state vector is $\mathbf{e}_{5}$, since this means the optimal strategy is to take the advantage of current good channel state before it expires so that the total cost can be minimized. On the other hand, if the current state is one of the "bad" states 1 or 2 , the results show that the best strategy is to wait as long as possible, in a hope that the channel turns better, since immediate transmission requires much more total cost for a successful transmission. Simulations indicate that in such case the waiting time in general tends to be the time until the channel distribution get close to stationary. If the current state is $\mathbf{e}_{3}$, the result shows that it is optimal to start transmission immediately, even though state 3 is not the target state. There are two reasons can account for this: First, the cost to transmit in state 3 takes $45 \%$ more power compared to state 4 for a successful delivery, but this is a small amount compared to

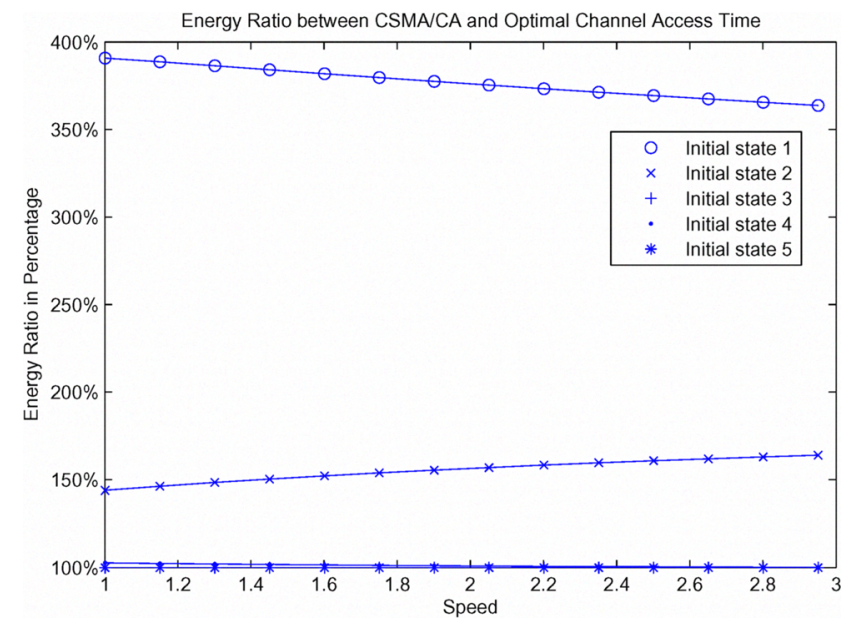

Fig. 5. Energy Comparison between CSMA/CA and Optimal Channel Access Time

the extra power required to transmit in state 1 and 2, which is $156 \%$ and $742 \%$ extra respectively. Secondly, as we can see from Fig. 2 and Fig. 3, for $\bar{\gamma}=5 \mathrm{~dB}$, if the initial state is $\mathbf{e}_{3}$, the probability of the channel being in state 4 and 5 increases rapidly. Thus immediate transmission of the entire frame can actually benefit from this. On the other hand, if the transmitter delays the transmission by waiting until the channel distribution gets close to stationary, the average energy cost could then become larger, since state 1 and state 2 will thus have more impact on the total cost.

Another thing is that if the starting state is given by vector $\mathbf{e}_{4}$, the result shows that the optimum access strategy changes from delay before transmit to immediate access. To see this, one needs to take the speed into consideration. First, an increase in a node's speed has the effect of increasing the convergence rate for a given initial state, as shown in Fig. 2 and Fig. 3. When the node's mobility is low, the probability of the channel being in state 4 or 5 can be very high in the long run, and from the weight vector we see that state 5 needs a lot less power for a successful transmission. Hence the node should rather wait before starting the transmission. However, when the node's mobility increases, the probability of the channel turning into a worse state quickly increases. This can result in a huge increase in energy consumption, and the best strategy is to take advantage of the current good state and start transmission immediately.

In Fig. 5, we show the energy ratio (in percentage) which is defined to be the ratio between energy spent by using the conventional CSMA/CA and by using the optimal channel access scheme proposed in this work. We can see that with initial state $\mathbf{e}_{1}$ and $\mathbf{e}_{2}$, the energy ratio is substantial, around $400 \%$ and $150 \%$ respectively. Especially, for starting state $\mathbf{e}_{1}$, CSMA/CA requires around $300 \%$ extra energy for one successful delivery compared the optimal case, which is a huge amount of cost. It clearly indicates that CSMA/CA performs very bad in a deep fade channel condition. We can however 
TABLE I

IEEE 802.15.4 MAC SUBLAYER CONSTANTS [5].

$$
\begin{aligned}
& \operatorname{macMinBE}=3 \\
& \text { aMaxBE }=5 \\
& \text { aUnitBackoffPeriod }=20 \quad \text { symbols } \\
& \text { aMaxFrameRetries }=3 \\
& \text { macAckWaitDuration }=120 \quad \text { symbols }
\end{aligned}
$$

see that with initial state $\mathbf{e}_{3}, \mathbf{e}_{4}$ and $\mathbf{e}_{5}$, there is almost no additional energy cost. This is because from the above, we already know that it is optimum to access the channel immediately, and this is almost the same as using CSMA/CA, only with a negligible (in the present context) time delay introduced by the backoff mechanism.

From above it is not hard to conclude that the actual energy performance of CSMA/CA depends very much on the channel condition at the time it starts the transmission. If the channel is unfriendly and node's mobility is low, then it is very likely that CSMA/CA will have a very bad energy performance and become the main reason for draining the node's battery fast. This drawback comes from the very design of the protocol, which is optimized to avoid packet collisions but doesn't address the physical channel condition the frame actually goes through.

\section{CONCLUSIONS}

In this paper, we first studied the widely applied CSMA/CA protocol, and used its application in the IEEE 802.15.4 standard as an example to show that CSMA/CA can be an very important source of energy waste in a low mobility, low traffic network, such as is the case in many WSN applications. The reason for this is that CSMA/CA was originally designed to avoid packet collisions, which is the dominating cause for transmission failures in a high traffic network. However, in a typical WSN, the traffic demand is much less, and a transmission failure is more likely to be caused by a deep channel fade rather than a collision. Therefore, we used a time-invariant Markov process to model the fading channel and formulated the design of an optimal MAC method as an optimization problem. The optimization revealed that the optimum channel access time can be very different from those used by currently standardized CSMA/CA protocols and that significant average energy savings may be achieved by optimizing access times with respect to the current channel state.

\section{APPENDIX}

Some of the default values used in ZigBee standard are listed in Table I.

\section{REFERENCES}

[1] G. Karlsson, S. Lindfors, M. Skoglund and G. Øien, "Cross-layer Optimization in short-range wireless sensor networks (CROPS) Technical Part". Available at: http://www.ee.kth.se/commth/projects/CROPS/doc.

[2] A. J. Goldsmith, S. B. Wicker, "Design Challenges for energyconstrained ad hoc wireless networks," IEEE Wireless Commun. Mag., Vol.9, No.4, pp. 8-27, Aug. 2002.
[3] J. F. Kurose and K. W. Ross, Computer Networking - A Top-Down Approach Featuring the Internet, Pearson Addison Wesley, 2005.

[4] Mustafa Ergen, "IEEE 802.11 Tutorial" available online at: http://wow.eecs.berkeley.edu/ergen/docs/ieee.pdf

[5] IEEE 802.15.4 Part 15.4: Wireless Medium Access Control (MAC) and Physical Layer (PHY) Specifications for Low-Rate Wireless Personal Area Networks (LR-WPANs). IEEE, Oct, 2003.

[6] IEEE 802.11. Part 11: Wireless LAN Medium Access Control (MAC) and Physical Layer (PHY) Specifications. IEEE, Aug, 1999.

[7] Sinem Coleri Ergen, "ZigBee/IEEE 802.15.4 Summary", available online at: http://pages.cs.wisc.edu/ suman/courses/838/papers/zigbee .pdf

[8] H. S. Wang, and N. Moayeri, "Finite-State Markov Channel - A Useful Model for Radio Communication Channels" IEEE Trans. Veh. Tech., Vol. 44, No.1, Feb. 1995.

[9] C. D. Meyer, Matrix Analysis and Applied Linear Algebra, SIAM, 2001

[10] S. Cui, A. Goldsmith, and A. Bahai, "Energy-Constrained Modulation Optimization," IEEE Transactions on Wireless Communications, Vol.4 No.5, pp. 2349-2360, Sept. 2005. 